\title{
An in vitro comparison of intraluminal biofilm bacteria transfer of three peripheral intravenous valved blood control catheters
}

\author{
Authors: Fidel Martinez-Gutierrez, Laura Boegli, \\ Alessandra Agostinho, Elpidio Morales Sánchez, \\ Horacio Bach, Facundo Ruiz, \& Garth James
}

NOTICE: This is a postprint of an article that originally appeared in American Journal of Infection Control on June 2013. http://dx.doi.org/10.1016/j.ajic.2013.03.252.

Ryder M, James G, deLancey Pulcini E, Parker A, "An in vitro comparison of intraluminal biofilm bacteria transfer of three peripheral intravenous valved blood control catheters," AJIC: American J Infection Control, June 2013: 41(6) Supplement: S127-S128 


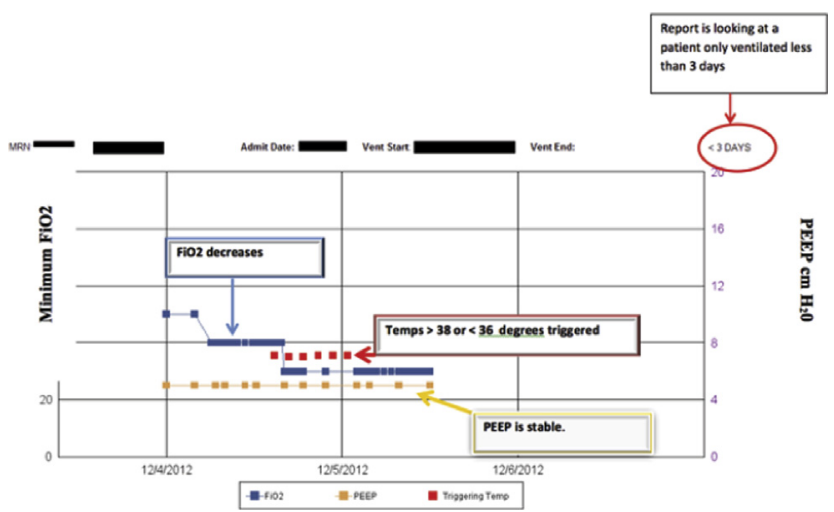

Figure 1. Example \#1 of data abstracted from the EPIC chart on a ventilated patient for daily VAE analysis. This patient was ventilated less than 3 days so would not meet criteria to review for a VAE. However, we chose to include these patients in a $<3$ day report so that Infection Prevention and the Critical care team could have an opportunity to intervene prior to a VAE. The graph casily demonstrates the decreasing FiO2 and stable PEEP line. The patient does have several triggers for fever but since neither the $\mathrm{FiO} 2$ nor the PEEP meet criteria, the infection practitioner does not need to evaluate further at this time.
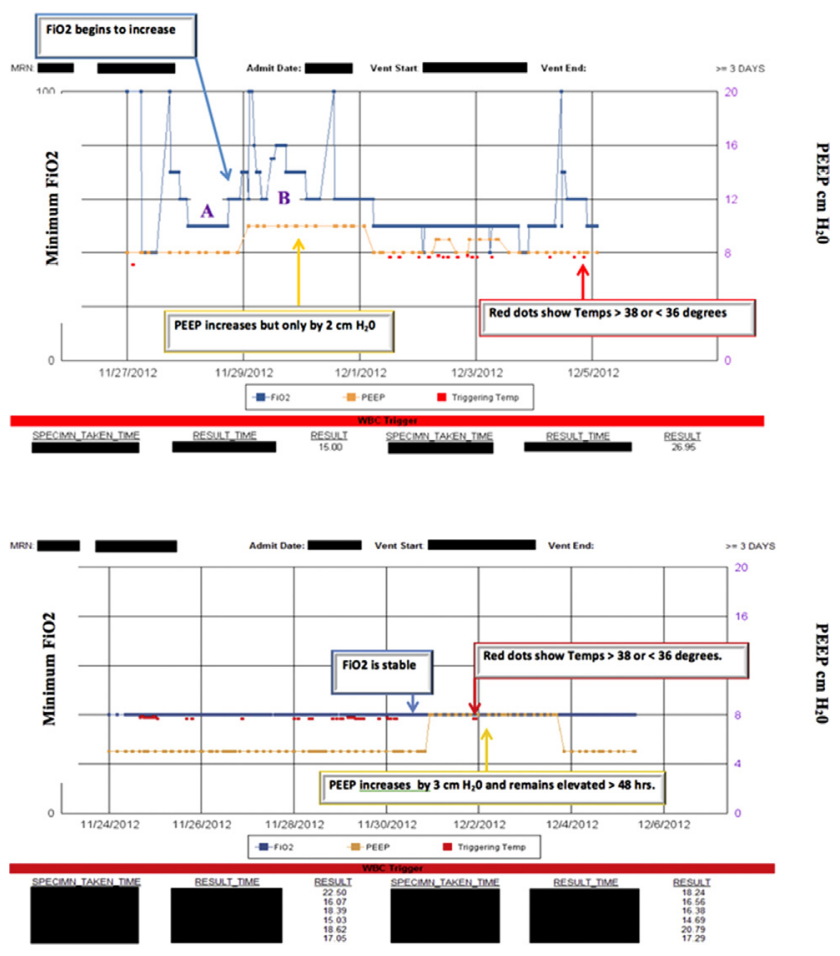

LESSON LEARNED: The EHR can be used to design reports that graphically display key pieces of clinical information allowing preventionists to quickly apply the new CDC VAE definition.

\section{Presentation Number 9-429}

Utilizing Interferon Gamma Release Assay Tests to Improve Surveillance for Latent Tuberculosis Infection in a Population at Risk

Catherine M. Lucas RN-BC, BSN, MS, CIC, Infection Prevention Coordinator, Canandaigua VA Medical Center, VA Healthcare Upstate New York Veterans Integrated Service Network 2; Elizabeth J. Davenport MT, Supervisory Medical Technologist, VAMC- Canandaigua; Cherisse S. Griffith BS, RPA-C, MBH-PA,
VAMC- Canandaigua; Christine J. Paris RN, AAS, BS, MBH-RN, VAMC- Canandaigua; Thomas Wong DO, Chief- Primary Care, VAMCCanandaigua

ISSUE: Our facility tuberculosis control program for patients, requires all patients be screened for latent Tuberculosis (TB) infection upon admission. The screening involves a two- step baseline Tuberculin Skin Test (TST). The time between the first and second TST is 1-3weeks. Compliance with the two -step TST proved challenging for our outpatient residential program, demonstrating an $80 \%$ failure rate for completion of the two- step TST from January 2011- January 2012. Contributing factors included returning to the clinic four times to complete the two- step TST for placement, interpretation, and recording results. This presented administrative and logistic challenges.

PROJECT: The project included admissions to an outpatient resi-dential program that serves Veterans with behavioral health and housing challenges. The Veterans admitted to the program have mental health disorders and substance abuse disorders. The program serves both male and female Veterans, from various Veteran eras across the age continuum. The project goal- improve compliance with Tuberculosis infection surveillance in our outpatient residential setting, improve safety for patients and staff and improve compliance with regulatory requirements by adopting IGRA testing for all admissions with routine admission lab work. Simplifying the testing process, would result in accurate results for latent TB infection. An educational program on Tuberculosis, facility TB risk assessment, and higher risk populations living in the residential outpatient setting was presented to leadership. The program helped define the inherent risk of a population at increased risk for latent TB infection. Leader-ship supported the change in testing. Working with our laboratory staff, supplies for IGRA testing and agreements with the testing laboratory were obtained. IGRA testing was started April, 2012 for admissions to our outpatient residential treatment program. RESULTS: Using IGRA tests inplace of a two - step tuberculin skin test resulted in greater than $95 \%$ compliance with admission screening for latent TB infection (LTBI) from April to November 2012. Screening with IGRA test and one visit to the lab instead of 4 clinic visits proved successful in eliminating barriers to compliance when screening for LTBI.

LESSON LEARNED: Using IGRA for latent TB infection surveillance in place of a two- step TST results in improved compliance, elimi-nating return to clinic appointments. IGRA testing adequately screens and identifies admissions with latent TB infection for public health reporting, quality assurance, and patient safety. Testing with IGRA on admission for a population at greater risk of TB infection provides consistent and reliable results. Simplifying the testing process eliminates most barriers.

Presentation Number 9-431

An In vitro Comparison of Intraluminal Biofilm Bacteria Transfer of Three Peripheral Intravenous Valved Blood Control Catheters

Marcia Ryder PhD, MS, RN, Research Scientist, Ryder Science, Inc; Garth James PhD, Associate Research Professor, Medical Biofilm Laboratory Manager, Center for Biofilm Engineering, Montana State University; Elinor deLancey Pulcini PhD, Assistant Research Professor, Medical Biofilms Laboratory, Center for Biofilm Engineering, Montana State University; Albert Parker PhD, Mathematics and Statistics, Center for Biofilm Engineering, Montana State University 


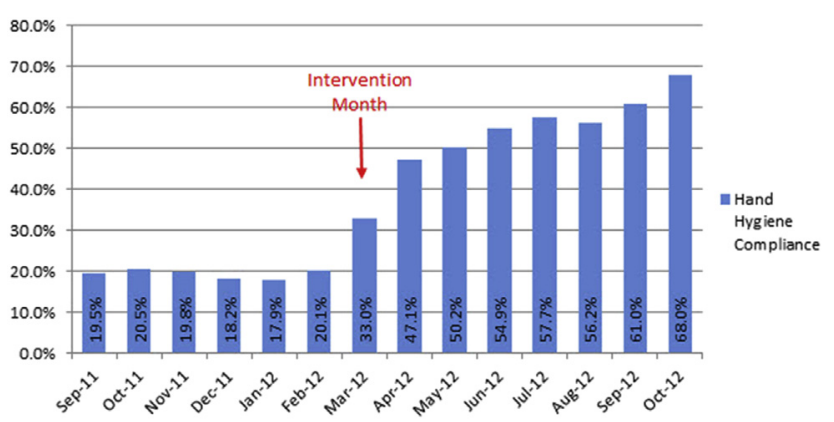

BACKGROUND/OBJECTIVES: The insertion of peripheral intravenous catheters (PIVC) is the most common invasive procedure performed by nurses. The new generation of PIVCs developed to reduce blood exposure during insertion utilizes additional internal components within the catheter hub. These components increase the internal surface area that is thought to increase biofilm formation and subsequent transfer of bacteria into the bloodstream. This raises concern for increased risk of bloodstream infection. The purpose of the this study is to compare biofilm formation and bacterial transfer rate between 3 valved blood control PIVCs in a clinically simulated in vitro model.

METHODS: The three PIVs tested were the Smiths Medical ViaValve ${ }^{\mathrm{TM}}$ Safety I.V. catheter (C1), the BD Insyte ${ }^{\mathrm{TM}}$ Autoguard ${ }^{\mathrm{TM}} \mathrm{BC}$ Shielded I.V. Catheter (C2) and the B.Braun Introcan Safety ${ }^{\circledR} 3$ catheter (C3). Six experiments were run with three time points measured within each run: 0,72 and 96 hours. Catheters were preconditioned with bovine serum albumin. A needleless connector was attached to each catheter, inoculated by flushing with $0.5 \mathrm{ml}$ of a 104 colony forming units per $\mathrm{ml}(\mathrm{CFU} / \mathrm{ml})$ of S. aureus, and incubated at room temperature for 2 hours. The connectors were then replaced with new sterile connectors and unattached bacteria were rinsed from the fluid path using sterile Phosphate Buffered Saline (PBS). Catheters were then either sampled or subjected to simulated clinical use by flushing 17 times daily with $0.5 \mathrm{ml}$ sterile nutrient and 1 flush at the end of the day with normal saline for 72 and 96 hours. Catheters were sampled with a two-step procedure. First, the assembly was flushed with $1.1 \mathrm{ml}$ PBS to recover planktonic and loosely attached bacteria and plated to determine $\mathrm{CFU} / \mathrm{ml}$ (Flush 1 ). Then the assemblies were surface disinfected, sonicated in PBS to remove firmly attached bacteria, and flushed a second time with $1.1 \mathrm{ml}$ PBS and plated (Flush 2).

RESULTS: When pooled across time points and all experiments, there were statistically significantly smaller bacterial mean log densities in Flush 1 and Flush 2 for the $\mathrm{C} 1$ catheter compared to the $\mathrm{C} 2$ ( $\mathrm{p}$-value $=0.003$ and 0.001 respectively) or C3 catheters ( $\mathrm{p}$ value $=0.014$ and 0.010 respectively)

CONCLUSIONS: There were statistically significantly fewer bacteria in flush samples from the $\mathrm{C} 1$ catheter compared to the other test catheters in a clinically simulated model. These results indicate that there are differences in the potential risk for transfer of bacteria into the bloodstream between different blood control valved PIVC designs.

\section{Presentation Number 9-432}

\section{The Effects of Executive Involvement, Goal Setting, Targeted Education and Caregiver Recognition on Hand Hygiene Performance}

Cheryl Bailey RN, BSN, MBA, CNO/Vice-President Patient Care Services, Cullman Regional Medical Center; Cheryl Bailey RN, BSN, MBA, CNO/Vice-President Patient Care Services, Cullman Regional Medical Center
BACKGROUND/OBJECTIVES: Based upon the challenges of improving and sustaining hand hygiene compliance through conventional means, increasing regulatory requirements and a desire to improve patient care, this 145 bed acute care Medical Center decided to implement and evaluate an automated hand hygiene monitoring system. The objective of the study was to measure the impact of automated hand hygiene monitoring system deployment on hand hygiene solution dispensing, hand hygiene compliance and infection rates before and after executive intervention. The initial implementation and application of technology alone, however, did not yield the desired results.

METHODS: This Medical Center installed a wireless hand hygiene monitoring system in a 31 bed medical-surgical unit. We monitored 62 employees including physicians, nurses, nursing assistants, physician assistants, clinical educators, as well as physical therapy and administrative staff. Hand hygiene solution dispensing, hand hygiene compliance and HAI rates were measured and reported from September 2011 through December 2012.

RESULTS: During a five week baseline period, the unit experienced a $51.3 \%$ increase in soap dispensing, a $37.3 \%$ increase in sanitizer dispensing, and a $45.2 \%$ increase in total hand hygiene solution dispensing. Although these were positive results, these gains did not result in a significant overall increase in hand hygiene compliance. It was only after increased executive involvement and targeted education as well as caregiver recognition that the compliance rates improved. The unit reported a 191.2\% improvement when comparing pre-intervention compliance (average $21.3 \%$ ) to postintervention compliance (average 56.4\%). Individual hand hygiene compliance rate increases for the employees were statistically significant (Students t-test, paired $\mathrm{t}=7.780369$ (49), $\mathrm{p}=4.1456$-E09, SAS). Average monthly hand hygiene solution dispenses increased by $91.2 \%$, and healthcare associated infections (HAIs) decreased by $25.0 \%$ during the post-intervention time period. These HAI reductions equate to a direct cost savings of $\$ 53,376$, a bottom line impact of $\$ 29,700$, and 49.2 days length of stay avoided.

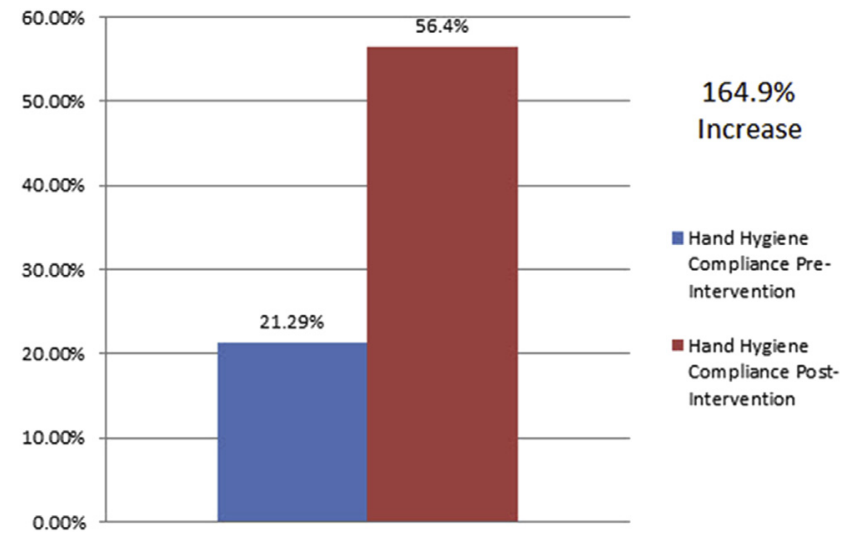

CONCLUSIONS: The Medical Center's implementation of an electronic hand hygiene monitoring system was most effective after increased executive involvement, goal setting, targeted education, and caregiver recognition. The combination of these elements enhanced caregiver hand hygiene performance and resulted in improved clinical and financial outcomes. The initial improvements were best achieved only after the technology was paired with implementation of an "Executive Push Package" that included clear executive support, visible leadership involvement, goal setting and a caregiver awareness and engagement campaign. 